Effect of L-carnitine administration on the seminal characteristics of Pietrain boars

A. Pruneda ${ }^{1}$, E. Pinart ${ }^{1}$, M.D. Briz ${ }^{1}$, S. Sancho ${ }^{1}$, E. Bussalleu ${ }^{1}$, M. Yeste ${ }^{1}$, I. Casas ${ }^{1}$, A. Fàbrega ${ }^{1}$, X. Barrera ${ }^{2}$, G. Mas ${ }^{3}$, S. Bonet ${ }^{1}$

${ }^{1}$ Group of Animal and Human Reproduction, University of Girona, Spain

${ }^{2}$ Semen Cardona S.L., Spain

${ }^{3}$ UPB España S.A., Spain

Dietary supplementation with L-carnitine increased sperm concentration and sperm motility in some species. In mammals, L-carnitine is highly concentrated in the epididymis and spermatozoa, where it may serve as an intramitochondrial vehicle for the acyl group, which in the form of acyl CoA acts as a substrate for the oxidative process producing energy for sperm motility. The objective of this experiment was to test the hypothesis that L-carnitine supplementation improves the semen characteristics of Pietrain boars. The study was performed using 12 healthy post-pubertal 10month-old Pietrain boars. Six boars were fed the control diet (a fortified corn and soybean meal-based diet) and six the control diet plus L-carnitine $(250 \mathrm{mg} / \mathrm{d})$. Semen was collected twice a week; conventional semen analyses were performed on weeks $0,2,4,6,8$, and 10. Sperm concentration, sperm motility and sperm morphology were analysed with a sperm computerassisted analyzer. Sperm integrity was evaluated using a fluorochrome multistaining method. The results are presented as means \pm S.E.M.; differences were analyzed with two-way ANOVA (with main effects of treatment and time), and $P<0.05$ was used for statistical significance.

Oral administration of $250 \mathrm{mg}$ L-carnitine/d for 10 weeks did not affect sperm volume, sperm concentration, or sperm integrity $(P>0.05)$ in Pietrain boars. However, supplementation with L-carnitine had a positive effect on sperm morphology, due to an increase of the frequency of morphologically normal spermatozoa $(P \leq 0.01 ; 69.17 \pm 11.51$ and $93.55 \pm 3.04$ for control and treated boars in the 10th week) associated with a decrease in the frequency of spermatozoa with distal droplets $(P \leq 0.01 ; 26.20 \pm 5.77$ and $1.50 \pm 0.60$ for control and treated boars in the 10th week). Progressively motile sperm were also higher in boars supplemented with L-carnitine $(35.27 \pm 7.75$ and $46.52 \pm 9.31$ for control and treated boars in the 10th week; $P=0.15$ ).

Based on these findings, we inferred that L-carnitine acts at an epididymal level, facilitating the release of the distal droplet and improving the kinetics of spermato- zoa. However, more functional analysis of spermatozoa are needed to better understand the effect of L-carnitine on spermatozoa. Further experiments with various concentrations of oral-administered L-carnitine should also be performed. Due to the lack of differences in sperm concentration, we concluded that oral administration of L-carnitine did not increase testicular production of sperm by Pietrain boars.

DOI: 10.1016/j.theriogenology.2008.06.044

\section{Seminal vesicle fluid reduces binding of porcine epididymal spermatozoa to oviduct explants}

P.M. Summers, S.T. Pena Jr.

School of Veterinary and Biomedical Sciences, James Cook University, Townsville 4811, Australia

Objective: The purpose was to determine if exposure of porcine epididymal sperm to seminal vesicle fluid (SVF) would influence the number of sperm bound to oviduct explants. Study design and methods: Testes and seminal vesicles were obtained from five 12 month-old Large White boars at slaughter. Sperm were collected from the rete testis, caput, corpus, and cauda epididymidis; an aliquot $(50 \mu \mathrm{L})$ was suspended in $1 \mathrm{ml}$ of SVF and incubated at $39^{\circ} \mathrm{C}$ for either 1 or $30 \mathrm{~min}$. For controls, sperm samples were incubated in Androhep medium (Petrunkina AM, et al. Reproduction 2001;121:889-96). After incubation, the sample was centrifuged at $600 \times g$ for $10 \mathrm{~min}$ and sperm suspended in modified Androhep medium at $5 \times 10^{6} \mathrm{~mL}^{-1}$. Before and after incubation in SVF, the capacitation status was determined by the chlortetracycline assay and viability by eosin-nigrosin staining. Oviduct explants were prepared from the isthmus and ampulla from non-cycling 20-week-old gilts using procedures modified from previous reports (Petrunkina AM, et al. Reproduction 2001;121:889-96; Suarez SS, et al. Biol Reprod 1991;56:447-53). Oviduct explants were set up as six replicates for each sperm sample and after incubation with sperm for $15 \mathrm{~min}$ at $39{ }^{\circ} \mathrm{C}$ in $5 \% \mathrm{CO}_{2}$ in air, the explants were fixed, stained and number of sperm bound in area of $1.25 \mathrm{~mm}^{2}$ counted. Data were analysed with ANOVA. Results: There was no significant difference in the number of sperm that bound to explants after incubation for $1 \mathrm{~min}$ in either Androhep medium or SVF, nor was there any difference in sperm viability (average 90\% viability), but SVF significantly reduced the percentage of uncapacitated sperm (70\% vs. $80 \%)$. After incubation for $30 \mathrm{~min}$ in SVF, fewer sperm $(P \leq 0.05)$ from the cauda and 
corpus, but not the caput or rete testis, bound to both isthmus and ampulla explants. The number that bound to the isthmus and ampulla explants was $6.23 \pm 0.87$ and $4.0 \pm 0.47$, respectively, whereas the controls were $13.13 \pm 1.74$ and $6.65 \pm 0.68$. There was no significant difference between the treatments for sperm viability, but the percentage of uncapacitated sperm declined significantly after incubation in SVF compared to the control (35\% vs. $60 \%$ for caudal sperm). Conclusion: The induction of capacitation by exposure to SVF was responsible for the reduced binding of sperm from the corpus and cauda to oviduct explants. This effect was not evident in sperm from the caput or rete testis. It was suggested that seminal plasma increases the binding of caudal sperm to the isthmus (Petrunkina AM, et al. Reproduction 2001;121:889-96); perhaps in vivo, components of seminal plasma other than SVF counter the capacitating effect, and add molecules to sperm to facilitate binding to the oviductal epithelium.

\section{DOI: $10.1016 /$ j.theriogenology.2008.06.045}

\section{The effect of glove type on boar semen quality}

Basim J. Awda, Mary M. Buhr

Department of Animal \& Poultry Science, University of Guelph, ON, Canada

The gloved hand method is commonly used to collect semen from boars. In this study, the effects of latex and non-latex (vinyl) gloves on sperm motility and viability were examined (one collection from each of four boars for each glove type). The sperm-rich fraction of the ejaculates were collected in a $35^{\circ} \mathrm{C}$ thermos, with sperm allowed to be in contact with gloves during collection. After collection, semen was extended in BTS buffer ( $\mathrm{pH} 7.3-7.4 ; 1 \times 10^{7}$ spermatozoa/mL) and assessed for sperm motility using CASA and viability using PI and SYBR-14 dyes, immediately and after incubation for $30 \mathrm{~min}$ at $36{ }^{\circ} \mathrm{C}$. There were differences $(P<0.05)$ between latex and non-latex gloved hand methods. Latex gloves significantly reduced the initial total sperm motility (TM), progressive velocity (VSL), and track speed (VCL); after 30 min incubation, only TM and VCL were significantly reduced. However, sperm viability was not significant different between the two kinds of gloves. We concluded that latex gloves had detrimental effects on TM, VSL, and VCL, but not viability, and therefore they should be not use for semen collection.

DOI: $10.1016 /$ j.theriogenology.2008.06.046
Comparison of AutoMate ${ }^{\circledR}$ and the gloved-hand method for boar semen collection

Steve Terlouw ${ }^{1}$, Christian Simmet ${ }^{2}$, Tom Schlimgen ${ }^{1}$, John Schenk ${ }^{1}$, Eric James ${ }^{1}$, Gordon Gunderson ${ }^{1}$,

Brad Didion ${ }^{1}$, John Dobrinsky ${ }^{1}$

${ }^{1}$ International Center for Biotechnology, Minitube of America, Verona, WI, USA

${ }^{2}$ Minitüb, Tiefenbach, Germany

Introduction: A substantial portion of the cost of a terminal sire semen dose is attributed to labor. Therefore, commercial boar studs continually look for ways to reduce labor costs. The automated boar semen collection system, AutoMate ${ }^{\circledR}$, reduces the amount of labor required for boar semen collection. AutoMate works with an artificial cervix (AC), which provides a firm texture and grip for the boar's penis and a hygienic double sheath plastic liner for the separate collection of the preseminal liquid and the ejaculate. The objective of this trial was to compare the AutoMate and gloved-hand boar collection systems regarding collection efficiency, semen production, and sperm quality. Materials and methods: Nine boars representing three lines and three age groups $(9,18$, and 30 months) and trained for gloved-hand and AutoMate semen collection were used. Boars were collected on Monday and Thursday, alternating between AutoMate and gloved-hand at each collection. Time recording began upon entry into the collection chute, with a record of when the boar began thrusting and when ejaculation began and ended. Full ejaculates, less the presperm fractions, were weighed to record volume. SpermVision ${ }^{\circledR}$ was used to determine concentration and motility values. Bacterial swabs of the gloved-hand, $\mathrm{AC}$, and neat semen were streaked on blood agar plates immediately after semen collection. Colonies were counted $24 \mathrm{~h}$ after incubation at $37^{\circ} \mathrm{C}$. Data were analyzed using SAS (SAS Institute, Cary, NC, USA) Proc GLM for the main effects of boar, collection system and boar $\times$ collection system. Results and discussion: There were no significant differences between AutoMate and gloved-hand collection of boar semen for any variable measured. Boars responded similarly to AutoMate and gloved-hand collection systems as shown by time from chute entry to beginning of ejaculation $(2.7 \pm 0.13 \mathrm{~min}$ vs. $2.6 \pm 0.13 \mathrm{~min} ; P=0.3653)$ and ejaculation time ( $7.1 \pm 0.23 \mathrm{~min}$ vs. $7.2 \pm 0.23 \mathrm{~min} ; P=0.8031)$. Boars collected on the AutoMate compared to the gloved-hand system produced a similar volume $(230 \pm 5.7 \mathrm{~mL}$ vs. $223 \pm 5.7 \mathrm{~mL} ; P=0.369)$ and concentration $(0.28 \times$ $10^{9} \pm 0.02 \mathrm{~mL}^{-1}$ vs. $0.26 \times 10^{9} \pm 0.02 \mathrm{~mL}^{-1} ; \quad P=$ 0.4281). Semen motility from AutoMate $(88.2 \%)$ collections was not different from gloved hand 